\title{
Genetic Variants in ASCT2 Gene are Associated with the Prognosis of Transarterial Chemoembolisation-Treated Early- Stage Hepatocelluar Carcinoma
}

\author{
Nai-Jian Ge ${ }^{1 \&}$, Zhi-Yong Shi ${ }^{1,2 \&}$, Xiao-He Yu ${ }^{1}$, Xiao-Jun Huang ${ }^{3}$, You-Sheng Wu ${ }^{3}$, \\ Yuan-Yuan Chen ${ }^{4}$, Jin Zhang ${ }^{3}$, Ye-Fa Yang ${ }^{1 *}$
}

\begin{abstract}
Background: Hepatocellular carcinoma (HCC) is one of the most prevalent malignancies worldwide. Transarterial chemoembolisation (TACE) is the standardized therapy for intermediate stage HCC. However, the prognosis for HCC patients treated by TACE greatly varies. Thus, there is a critical need for finding biomarkers to predict the prognosis of HCC patients. The amino acid transporter-2 (ASCT2) is involved in tumorigenesis and progression of many malignancies. This study aimed to evaluate the predictive role of two single nuclear polymorphisms (SNPs, rs3826793 and rs2070246) in the ASCT2 gene in HCC patients treated by TACE. Materials and Methods: Two functional SNPs (rs3826793 and rs2070246) in the ASCT2 gene were selected and genotyped using the Sequenom iPLEX genotyping system in a cohort of 448 unresectable Chinese HCC patients treated by TACE. Univariate and multivariate Cox proportional hazards models and Kaplan-Meier curves were used for the prognosis analyses. Results: There was no significant association between two SNPs (rs3826793 and rs2070246) in the ASCT2 gene and overall survival of TACE treated HCC patients. However, we demonstrated that patients with early stage HCC carrying T genotype in rs2070246 showed better OS than those carrying CC genotype $(P=0.023)$. Conclusions: We demonstrated that patients with early stage $\mathrm{HCC}$ carrying $\mathrm{T}$ genotype in rs2070246 showed better OS than those carrying CC genotype.
\end{abstract}

Keywords: Hepatocellular carcinoma - ASCT2 gene - association study - prognosis - transarterial chemoembolisation

Asian Pac J Cancer Prev, 16 (9), 4103-4107

\section{Introduction}

Hepatocellular carcinoma (HCC) is one of the most prevalent malignancies worldwide. Recent studies show that the incidence and mortality rates of HCC are increasing (Jemal et al., 2010). Most patients with HCC are diagnosed at advanced stages of disease, where curative treatments, such as hepatic resection, liver transplantation and percutaneous ablation, are not feasible (Bruix et al., 2011). In clinical practice, transarterial chemoembolization (TACE) is considered the standard palliative trefatment for HCC patients at intermediate stage of the disease (Llovet et al., 2002; Llovet and Bruix, 2003). However, the prognosis for HCC patients treated by TACE varies greatly according to disease status. Up to date, clinical parameters like tumor size, portal invasion, performance status, and ChildPugh score, as well as laboratory parameters including bilirubin and alpha fetoprotein (AFP), have been reported as pretherapeutic prognostic and predictive parameters (Kohles et al., 2012). However, these parameters are not always accepted in routine clinical practice for estimation of prognosis. Therefore, it is extremely urgent to explore novel biomarkers to discriminate patient groups with different clinical outcomes and direct the treatment for HCC patients.

Amino acids are the primary source of cellular nitrogen, used for nucleotide, glutathione, amino sugar, and protein synthesis. Compared to normal cells or tissues, cancer cells display enhanced amino acid intaking and metabolism to support cell proliferation (Mazurek and Eigenbrodt, 2003). Comparing to other amino acid, cancer cells depend far more on gluatamine. Glutamine metabolism has been proposed to be an important metabolic phenotype of proliferating cancer cells. Previous cell culture studies and tumor implantation studies have shown that glutamine plays an important role in cancer cell growth and survival by contributing to ATP synthesis, as well as by providing intermediates for macromolecular synthesis (Reynolds et al., 2014). Moreover, reductive carboxylation of glutamine is critical for the metabolic reprogramming that enables

${ }^{1}$ Department of Radioactive Intervention, Eastern Hepatobiliary Surgery Hospital, Second Military Medical University, Shanghai, ${ }^{2}$ Soochow University, Suzhou, Jiangsu, ${ }^{3}$ State Key Laboratory of Cancer Biology and Experimental Teaching Center of Basic Medicine, Fourth Military Medical University, Xi'an, Shaanxi, ${ }^{4}$ Department of Ophthalmology, First People of Yulin, Yulin, China ${ }^{\&}$ Equal contributors *For correspondence: yangyefa55@163.com 
cancer cells to survive and proliferate under hypoxia (Mullen et al., 2014). And Glutamine uptake in many cancer cell lines is mediated primarily through Amino acid transporter-2 (ASCT2).

ASCT2 is a Na+-coupled amino acid transporter that belongs to SLC1 protein family. In spite of its dependence on $\mathrm{Na}+$, ASCT2 functions as an obligatory amino acid exchanger rather than an influx transporter. It accepts only neutral amino acids as substrates for transport in both direction. Glutamine is a preferred substrate of ASCT2. Increased expression of ASCT2 has been documented in several types of primary tumors. And it is essential for tumor cell growth and survival in cancer cell lines. However, to date, the association between ASCT2 and clinical outcome has not been investigated in HCC patients. Single nucleotide polymorphisms (SNPs) represent the most common form of genetic diversity within a species and account for much of the variation in genetic traits between patients (Shastry, 2009), including disease susceptibility, prognosis and response to therapy. In addition to amino acid change, SNPs directly affect gene functions through various translational or posttranslational mechanisms, such as altering miRNA binding, protein folding, the spliceosome formation or mRNA stability (Chamary et al., 2006). Whether genetic variants in ASCT2 gene can predict the prognosis of advanced HCC patients is unknown.

In this study, we assessed the associations of two single nuclear polymorphisms (SNPs) rs3826793 and rs2070246 in ASCT2 gene with clinical parameters and overall survival (OS) in a Chinese cohort of 448 advanced HCC patients treated by TACE. To the best of our knowledge, this is the first study to investigate the predictive role of SNPs in ASCT2 gene on HCC.

\section{Materials and Methods}

\section{Study population}

A total of 493 Han Chinese patients with unresectable HCC were recruited at the Department of Radioactive Intervention of Eastern Hepatobiliary Surgery Hospital, Second Military Medical University, in Shanghai, China between February 2008 and December 2011. All patients had no previous history of other cancers or cancer-related treatment, and were newly diagnosed as HCC by imaging technologies, laboratory examination and clinical data. There were no age, gender and stage restrictions for patient recruitment. TACE was used as the first-line treatment for all patients. In this study, we excluded 45 patients, including 32 with incomplete clinical data or failed follow up and 13 with poor quality of DNA. Finally, a total of 448 patients were included in the present study and successfully genotyped. This study was approved by the Ethic Committee of the Second Military Medical University, and signed informed consent was obtained from each participant.

\section{Demographic and clinical data}

Demographic data were collected through inperson interviews using a standardized epidemiological questionnaire, including age, gender, ethnicity, residential region, smoking status, alcohol use, education status, body mass index, and family history of cancer. Detailed clinical information was collected through medical chart review or consultation with treating physicians, including time of diagnosis, time of first TACE treatment, tumor size, number of lesions, tumor stage, location site, lymph node invasion, HBV-surface antigen (HBsAg) status, portal vein tumor thrombus (PVTT), and level of serum AFP. A standard follow-up was performed by a trained clinical specialist through on-site interview, direct calling, or medical chart review. The latest follow-up data in this analysis were obtained in June 2013. For all enrolled patients, $5 \mathrm{ml}$ of blood sample from each participant was collected and subjected for genomic DNA extraction using the E.Z.N.A. Blood DNA Midi Kit (Omega BioTek, Norcross, GA).

\section{TACE procedure}

The chemoembolization procedure consisted of embolization of the tumour-feeding arteries using lipiodol (Lipiodol Ultra-Fluide; Guerbet Laboratories, Aulnay-Sous-Bois, France) and sponge gelatin (Gelfoam; Hangzhou Alc, China; 350-560 $\mu \mathrm{m}$ indiameter), accompanied by chemotherapy with epirubicin (20-40 mg; Pharmorubicin; Pfizer,Wuxi, China). Patients were routinely injected with $2-20 \mathrm{ml}$ of lipiodol $(1-2 \mathrm{ml} / \mathrm{cm}$ diameter of the tumour). Lipiodol and gelatin sponges were used, alone or in combination, to occlude the tumour-feeding artery, according to individual conditions. Embolization was performed with absorbable gelatin sponge particles to achieve stasis in the tumour-feeding artery.In each case, the decision to repeat TACE was based on computed tomography (CT) or magnetic resonance imaging (MRI) results.

\section{SNP selection and genotyping}

Functional SNPs on the ASCT2 gene was selected using a set of Web-based SNP selection tools (freely available at http://snpinfo.niehs.nih.gov/snpinfo/snpfunc. htm), by which one can select SNPs based on linkage disequilibrium and predictfunctional characteristics of both coding and noncoding SNPs. Two functional SNPs on the ASCT2gene, rs3826793 and rs2070246, with $>5 \%$ minor allele frequency in Chinese population were selected for genotype assay. Among them, SNP rs3826793 is located in the 3'UTR region of ASCT2 gene, which may affect microRNA binding to ASCT2 mRNA. Another SNP rs2070246 located in the intron of ASCT2 gene may affect the splicing of mRNA. Genotyping was carried out using the iPLEX genotyping system (Sequenom, San Diego, CA). Laboratory persons who conduct genotyping assays were blinded to patients' information. The average call rate for the SNP assays was $99.3 \%$. Strict quality controls were implemented during genotyping, with $>99.0 \%$ concordance.

\section{Statistical analysis}

The SPSS Statistics 19.0 software (IBM) was used for all statistical analyses. Overall survival (OS) was defined as the period from the first TACE treatment to the date of death or last follow-up. The three genetic models 
Genetic Variants in ASCT2 Gene are Associated with Prognosis of TACE-Treated Early-Stage HCC

(additive, dominant and recessive) were applied to assess the association of SNPs with clinical outcome of HCC patients by univariate and multivariate Cox proportional hazard analyses. Kaplan-Meier survival curves and logrank test were used to assess the differences of OS among different subgroups. All $\mathrm{P}$ values were two-sided, and $P<0.05$ was considered statistically significant.

\section{Results}

Clinical characteristics and their association with the OS of HCC patients

This study included 448 unresectable HCC patients with first-line TACE treatment and the characteristics of these patients were summarized in Table 1 . The median age at the time of diagnosis was 54 years (range, 27-82 years), and $391(87.3 \%)$ patients were men. There were $385(85.3 \%)$ patients with positive serum HBsAg and 243 (54.2\%) patients who had a serum AFP level > 200ng/ $\mathrm{mL}$. The majority of patients $(70.1 \%)$ had a tumor size of $>5 \mathrm{~cm}$ and $58.9 \%$ had multiple lesions. There was almost equally distribution of BCLC stage B and C (49.4\% vs. $50.7 \%)$. The majority of patients $(70.1 \%)$ were absent of PVTT. During the median follow-up of 11 months (ranging from 1 to 78 months), 339 patients died of HCC. In multivariate Cox regression analysis adjusted by age, gender, HBsAg, serum AFP, tumor size and BCLC stage, significantly increased death risk was observed in patients with high level of serum AFP (HR, 1.35, 95\%CI 1.07-1.69, $\mathrm{P}=0.010)$, larger tumor diameter (HR, 1.94, 95\%CI 1.45-2.59, $P<0.0001$ ), PVTT (HR, $1.83,95 \%$ CI 1.42-2.34, $\mathrm{P}<0.0001)$ and with high stage $(\mathrm{HR}, 1.53$, 95\% CI 1.19-1.97, $P=0.001)$.

SNPs in ASCT2 gene showed no association with clinical outcomes of HCC

We assessed the associations between either of the two SNPs and OS of HCC patients suing univariate and multivariate Cox proportional hazard analysis. As showed in Table 2, in three genetic models, there was no significant association between genetic variants in ASCT2 gene and the clinical outcome of HCC. Similarly, Kaplan-Meier curve analysis also showed no difference between patients with different genoytpes (Figure 1). We then conducted
A

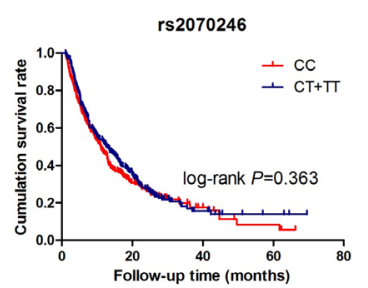

B

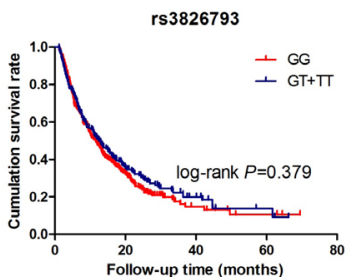

Figure 1. Kaplan-Meier Survival Curve of 448 HCC

Patients in two SNP. Kaplan-Meier survival curves showed no difference between patients with different genoytpes (A

Table 1. Characteristics of Study Population by Event Distributions

\begin{tabular}{|c|c|c|c|c|}
\hline varibles & alive, $n=102$ & death, $n=346$ & $\operatorname{HR}(95 \% \mathrm{CI})^{\mathrm{a}}$ & $P$-value \\
\hline \multicolumn{5}{|l|}{ Gender } \\
\hline Female & $16(15.7)$ & $41(11.8)$ & Ref. & \\
\hline Male & $86(84.3)$ & $305(88.2)$ & $1.14(0.82-1.59)$ & 0.422 \\
\hline \multicolumn{5}{|l|}{ Age, year } \\
\hline$<53$ & $56(54.9)$ & $180(52.0)$ & Ref. & \\
\hline$\geq 53$ & $46(45.1)$ & $166(48.0)$ & $1.05(0.84-1.31)$ & 0.688 \\
\hline \multicolumn{5}{|l|}{ HBsAg } \\
\hline Negative & $14(13.7)$ & $51(14.7)$ & Ref. & \\
\hline Positive & $88(86.3)$ & $295(85.3)$ & $0.89(0.65-1.22)$ & 0.474 \\
\hline \multicolumn{5}{|l|}{ AFP, ng/ml } \\
\hline$<200$ & $60(58.8)$ & $145(41.9)$ & Ref. & \\
\hline$\geq 200$ & $42(41.2)$ & $201(58.1)$ & $1.40(1.12-1.75)$ & 0.003 \\
\hline \multicolumn{5}{|l|}{ Tumor size, $\mathrm{cm}$} \\
\hline$<5$ & $52(51.0)$ & $82(23.7)$ & Ref. & \\
\hline$\geq 5$ & $50(49.0)$ & $264(76.3)$ & $2.07(1.58-2.70)$ & $<0.0001$ \\
\hline \multicolumn{5}{|l|}{ Tumor number } \\
\hline Single & $54(52.9)$ & $130(37.6)$ & Ref. & \\
\hline multiple & $48(47.1)$ & $216(62.4)$ & $1.37(1.10-1.71)$ & 0.006 \\
\hline \multicolumn{5}{|l|}{ PVTT } \\
\hline No & $86(84.3)$ & $228(65.9)$ & Ref. & \\
\hline Yes & $16(15.7)$ & $118(34.1)$ & $2.31(1.82-2.94)$ & $<0.0001$ \\
\hline \multicolumn{5}{|l|}{ TNM stage } \\
\hline early stage (I) & $54(52.9)$ & $268(77.5)$ & Ref. & \\
\hline late stage (II-IV) & $48(47.1)$ & $78(22.5)$ & $2.33(1.79-3.03)$ & $<0.0001$ \\
\hline \multicolumn{5}{|l|}{ BCLC stage } \\
\hline B stage & $83(81.4)$ & $201(58.1)$ & Ref. & \\
\hline C stage & 19 (18.6) & $145(41.9)$ & $2.28(1.81-2.86)$ & $<0.0001$ \\
\hline \multicolumn{5}{|l|}{ Combined therapy } \\
\hline No & $64(62.7)$ & $302(87.3)$ & Ref. & \\
\hline Yes & $38(37.3)$ & $44(12.7)$ & $0.43(0.31-0.60)$ & $<0.0001$ \\
\hline
\end{tabular}

a, Multivariate analyses were adjusted for age, gender, HBsAg, AFP and BCLC stage where appropriate 
Table 2. Association of SNPs with clinical outcome of HCC patients

\begin{tabular}{|c|c|c|c|c|c|c|}
\hline \multirow[b]{2}{*}{ SNP } & \multirow[b]{2}{*}{ Genotype } & \multicolumn{3}{|c|}{ Univariate analysis } & \multicolumn{2}{|c|}{ Multivariate analysisa } \\
\hline & & Death/Total & HR $(95 \% \mathrm{CI})$ & $P$ value & $\mathrm{HR}(95 \% \mathrm{CI})$ & $P$ value \\
\hline \multirow[t]{2}{*}{ rs3826793 } & GG & $203 / 264$ & Ref. & & Ref. & \\
\hline & $\mathrm{GT}+\mathrm{TT}$ & $129 / 167$ & $0.90(0.72-1.13)$ & 0.379 & $0.97(0.77-1.21)$ & 0.78 \\
\hline \multirow[t]{2}{*}{ rs2070246 } & $\mathrm{CC}$ & $171 / 213$ & Ref. & & Ref. & \\
\hline & $\mathrm{CT}+\mathrm{TT}$ & $174 / 233$ & $0.90(0.73-1.11)$ & 0.363 & $0.97(0.78-1.20)$ & 0.769 \\
\hline
\end{tabular}

a, Multivariate analyses were adjusted for age, gender, HBsAg, AFP and BCLC stage

Table 3. Stratified Analysis of the Association bettween SNP and Prognosis of HCC Patients

\begin{tabular}{ccc}
\hline rs3826793 & & rs2070246 \\
\cline { 2 - 3 }$(95 \% \mathrm{CI})$ a & $P$ value &
\end{tabular}

TNM stage

Early stage $\quad 0.97(0.68-1.37) \quad 0.379 \quad 1.72(1.07-2.75) \quad 0.023$

Late stage $\quad 0.99(0.74-1.33) \quad 0.384 \quad 1.08(0.82-1.44) \quad 0.936$

$*_{\mathrm{a}, \text { Multivariate analyses were adjusted for age, gender, } \mathrm{HBsAg}, \mathrm{AFP}}$ and BCLC stage
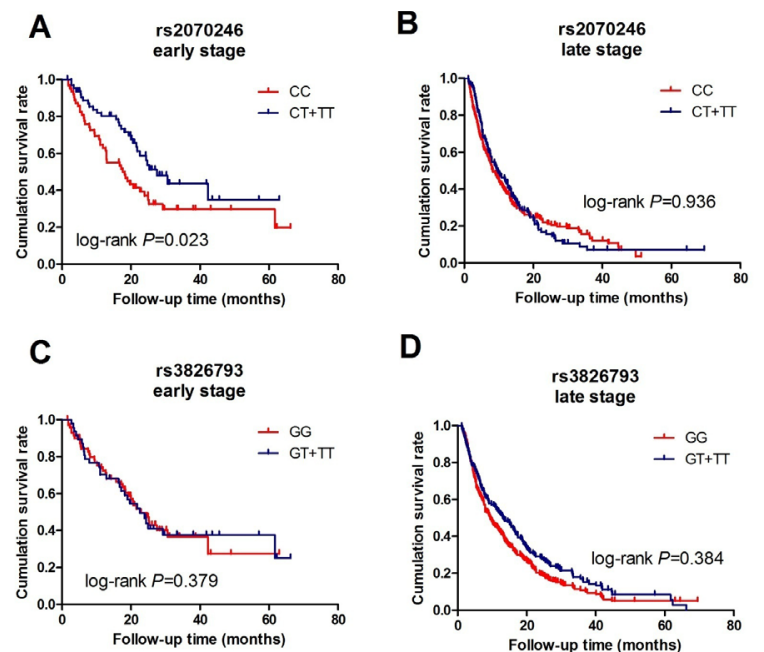

Figure 2. Kaplan-Meier plots of Overall Survival in Patients with Different Stage. A) Patients with early stage HCC carrying TT genotype in rs2070246 showed better OS than those carrying $\mathrm{CC}$ genotype $(P=0.023)$. B)There was no significant difference in the OS of late-stage $\mathrm{HCC}$ patients with different genotypes in $\operatorname{rs} 2070246(P=0.271)$. C and D) There was no significance in the OS of either early-stage or late-stage HCC patients with different genotypes in rs3826793.

the stratified analysis based on TNM stage of tumor. As shown in Table 3 , there was significant association of rs2070246 with the outcome of HCC patients in each stage. Kaplan-Meier curve analysis further demonstrated that patients with early stage HCC carrying T genotype in rs2070246 showed better OS than those carrying C genotype ( $P=0.023$, Figure $2 \mathrm{~A})$. Nevertheless, there was no significant difference in the OS of late-stage HCC patients with different genotypes in rs2070246 ( $P=0.271$, Figure 2B). Moreover, there was no significance in the OS of either early-stage or late-stage HCC patients with different genotypes in rs3826793 (Figure 2C and D).

\section{Discussion}

Only about $20 \%$ patients with HCC are suitable to surgical resection, which highlights the importance of non- surgical therapies. The beneficial effects of TACE have been shown by other studies, in particular, unresectable HCC patients are treated mainly with palliative TACE. Considering the fact that the outcome for individual HCC patient greatly varied, biomarkers were urgently needed to found, which can predict the outcome of the HCC patients. We choosed unresectable Chinese HCC patients as study population.

In the present study, we evaluated the effects of 2 functional SNPs in ASCT2 gene on the prognosis of a cohort of $448 \mathrm{HCC}$ patients. We demonstrated that in HCC patients with early stage tumor, the SNP rs2070246 was significantly associated with the OS. To the best of our knowledge, this is the first study to report that genetic variants in ASCT2 gene have a significant effect on the prognosis of patients with early stage HCC.

Glutamine is an abundant and versatile nutrient that participates in energy formation, redox homeostasis, macromolecular synthesis, and signaling in cancer cells (Hensley et al., 2013). As a major transporter of glutamine, ASCT2 plays an critical role in tumour growth and the proliferation of cancer cells (Fuchs and Colonna, 2006). It provides cancer cells with essential amino acids for protein synthesis, and it coordinates tumour cell growth through the activation of mammalian target of rapamycin (mTOR) (Fuchs et al., 2007). Previous studies have demonstrated that ASCT2 is highly expressed in various malignancies, including $\mathrm{HCC}$ and colorectal and prostate cancer, and its expression is closely associated with tumour aggressiveness and prognosis in colorectal or prostate cancer (Li et al., 2003; Fuchs et al., 2007; Namikawa et al., 2014).

Genetic variants such as SNPs play an important role in the regulation of gene expression, mRNA degradation and translation, as well as protein structures which may affect the biological functions of proteins (Shastry, 2009). Considering the critical role of ASCT2 in the progression of cancer, effects of SNPs in ASCT2 gene on cancer cell proliferation, invasion and drug sensitivity as well as clinicopathologcal characteristics and prognosis of patients should be investigated. However, little is known on the associations between these SNPs and cancer risk as well as patient prognosis. In this study, we found that SNP rs2070246 in ASCT2 was significantly associated with poor prognosis of $\mathrm{HCC}$ patients with early stage diseases. Although the molecular and cellular functions of this SNP have not yet been evaluated, silicon analysis found that rs2070246 may affect alternative splicing of mRNA, which may affect the structure and biological activity of ASCT2 protein, and ultimately affect the progression of HCC and survival of patients. However, we 
did not observe significant association of rs2070246 with the OS of patients with late stage diseases. These findings suggest that rs2070246 might affect the progression of early stage HCC. Once tumors progress into late stage, other oncogenic factors might compensate the effects of rs2070246. On the other hand, we did not observe significant association between SNP rs3826793 and the prognosis of HCC patients. Possible explanation for our results is that rs3826793 may not or just very weakly affect the expression and functions of ASCT2 in HCC. Nevertheless, all these hypotheses need further investigation in future study.

Our study has several strengths and limitations. The patient population is enrolled from Eastern Hepatobiliary Surgery Hospital with the extremely low rate of patient loss during follow-up, which is highly attractive in conducting population-based research. In addition, the homogenous patient characteristics and treatments greatly reduced the confounding effects of heterogeneous therapeutic modalities seen in many cancer clinical outcome studies. However, our sample size may not be large enough, and then a retrospective study is not as effective as perspective study. Therefore, further validation is needed for our results in future study. Furthermore, we did not examined the biological functions of these two SNPs, which need further experimental investigation in future.

In summary, as the first study observing the effect of ASCT2 gene polymorphisms on HCC

prognosis, our study strongly suggest that SNP rs2070246 in ASCT2 genes may be an independent prognostic marker for OS in HCC patients with early stage tumor. These findings warrant further studies on the impact of this SNP on the effectiveness of therapeutics against ASCT2 in HCC.

\section{Acknowledgements}

This work was supported by grants 81272522 from the National Natural Science Foundation of China.

\section{References}

Bruix J, Sherman M, American Association for the Study of Liver D (2011). Management of hepatocellular carcinoma: an update. Hepatology, 53, 1020-2.

Chamary JV, Parmley JL, Hurst LD (2006). Hearing silence: non-neutral evolution at synonymous sites in mammals. Nat Rev Genet, 7, 98-108.

Fuchs A, Colonna M (2006). The role of NK cell recognition of nectin and nectin-like proteins in tumor immunosurveillance. Semin Cancer Biol, 16, 359-66.

Fuchs BC, Finger RE, Onan MC, et al (2007). ASCT2 silencing regulates mammalian target-of-rapamycin growth and survival signaling in human hepatoma cells. Am J Physiol Cell Physiol, 293, 55-63.

Hensley CT, Wasti AT, DeBerardinis RJ (2013). Glutamine and cancer: cell biology, physiology, and clinical opportunities. J Clin Invest, 123, 3678-84.

Jemal A, Siegel R, Xu J, et al (2010). Cancer statistics, 2010. CA Cancer J Clin, 60, 277-300.

Kohles N, Nagel D, Jungst D, et al (2012). Prognostic relevance of oncological serum biomarkers in liver cancer patients undergoing transarterial chemoembolization therapy.

$$
\text { Tumour Biol, 33, 33-40. }
$$

Li R, Younes M, Frolov A, et al (2003). Expression of neutral amino acid transporter ASCT2 in human prostate. Anticancer Res, 23, 3413-8.

Llovet JM, Bruix J (2003). Systematic review of randomized trials for unresectable hepatocellular carcinoma: Chemoembolization improves survival. Hepatol, 37, 429-42.

Llovet JM, Real MI, Montana X, et al (2002). Arterial embolisation or chemoembolisation versus symptomatic treatment in patients with unresectable hepatocellular carcinoma: a randomised controlled trial. Lancet, 359, 1734-9.

Mazurek S, Eigenbrodt E (2003). The tumor metabolome. Anticancer Res, 23, 1149-54.

Mullen AR, Hu Z, Shi X, et al (2014). Oxidation of alphaketoglutarate is required for reductive carboxylation in cancer cells with mitochondrial defects. Cell Rep, 7, 167990.

Namikawa M, Kakizaki S, Kaira K, et al (2014). Expression of amino acid transporters (LAT1, ASCT2 and xCT) as clinical significance in hepatocellular carcinoma. Hepatol Res.

Reynolds MR, Lane AN, Robertson B, et al (2014). Control of glutamine metabolism by the tumor suppressor $\mathrm{Rb}$. Oncogene, 33, 556-66.

Shastry BS (2009). SNPs: impact on gene function and phenotype. Methods Mol Biol, 578, 3-22. 